\title{
Motion of particles on a four-dimensional asymptotically AdS black hole with scalar hair
}

\author{
P. A. González ${ }^{1, \mathrm{a}}$, Marco Olivares ${ }^{1, \mathrm{~b}}$, Yerko Vásquez ${ }^{2, \mathrm{c}}$ \\ ${ }^{1}$ Facultad de Ingeniería, Universidad Diego Portales, Avenida Ejército Libertador 441, Casilla 298-V, Santiago, Chile \\ ${ }^{2}$ Departamento de Física, Facultad de Ciencias, Universidad de La Serena, Avenida Cisternas, 1200 La Serena, Chile
}

Received: 7 September 2015 / Accepted: 18 September 2015 / Published online: 30 September 2015

(C) The Author(s) 2015. This article is published with open access at Springerlink.com

\begin{abstract}
Motivated by black hole solutions with matter fields outside their horizon, we study the effect of these matter fields on the motion of massless and massive particles. We consider as background a four-dimensional asymptotically AdS black hole with scalar hair. The geodesics are studied numerically and we discuss the differences in the motion of particles between the four-dimensional asymptotically AdS black holes with scalar hair and their no-hair limit, that is, Schwarzschild AdS black holes. Mainly, we found that there are bounded orbits like planetary orbits in this background. However, the periods associated to circular orbits are modified by the presence of the scalar hair. Besides, we found that some classical tests such as perihelion precession, deflection of light, and gravitational time delay have the standard value of general relativity plus a correction term coming from the cosmological constant and the scalar hair. Finally, we found a specific value of the parameter associated to the scalar hair, in order to explain the discrepancy between the theory and the observations, for the perihelion precession of Mercury and light deflection.
\end{abstract}

\section{Contents}

1 Introduction ...................

2 Four-dimensional asymptotically AdS black holes with scalar hair . . . . . . . . . . . . . . 2

3 Geodesics.................... 3

3.1 Time-like geodesic . . . . . . . . . . . . . . . . . 4

3.1.1 Radial motion . . . . . . . . . . . . . . 4

3.1.2 Angular motion ............ 4

3.1.3 Perihelion precession ......... 4

3.2 Null geodesic . . . . . . . . . . . 6

\footnotetext{
a e-mail: pablo.gonzalez@udp.cl

b e-mail: marco.olivaresr@mail.udp.cl

c e-mail: yvasquez@userena.cl
}

3.2.1 Radial motion . . . . . . . . . . . . . . 6

3.2 .2 Angular motion . . . . . . . . . . . 6

3.2.3 Deflection of light . . . . . . . . 7

3.2.4 Gravitational time delay ....... . . 8

4 Concluding comments . . . . . . . . . . . . 9

References . . . . . . . . . . . . . . . . . . 9

\section{Introduction}

Hairy black holes are interesting solutions of Einstein's Theory of Gravity and also of certain types of Modified Gravity Theories. The first attempts to couple a scalar field to gravity was done in an asymptotically flat spacetime finding hairy black hole solutions [1-3] but these solutions violated the nohair theorems because they were not physically acceptable as the scalar field was divergent on the horizon and stability analysis showed that they were unstable [4]. Then, by introducing a cosmological constant hairy black hole solutions with a minimally coupled scalar field and a self-interaction potential in asymptotically dS space were found, but they were unstable $[5,6]$. Also, a hairy black hole configuration was reported for a scalar field non-minimally coupled to gravity [7], but perturbation analysis showed the instability of the solution $[8,9]$. In the case of a negative cosmological constant, stable solutions were found numerically for spherical geometries $[10,11]$ and an exact solution in asymptotically AdS space with hyperbolic geometry was presented in [12] and generalized later to include electric charge $[13,14]$. Then a generalization to non-conformal solutions was discussed in [15]. Further hairy solutions in the presence of a cosmological constant were reported in [16-21] with various properties. On the other hand, by introducing a coupling of a scalar field to Einstein tensor that acts as an effective cosmological constant $[22,23]$ a hairy black hole solution was presented [24], and spherically symmetric hairy black hole solutions with scalar hair were found [25]. Additionally, there 
are also very interesting recent developments in Observational Astronomy. High precision astronomical observations of the supermassive black holes may pave the way to experimentally test the no-hair conjecture [26]. Also, there are numerical investigations of single and binary black holes in the presence of scalar fields [27], and more recently it was shown that it is not necessary to introduce a cosmological constant to get scalar hairy black holes [28,29].

On the other hand, the recent developments in string theory and specially the application of the AdS/CFT principle to condensed matter phenomena like superconductivity (for a review see [30]), triggered the interest of further study of the behavior of matter fields outside the black hole horizon $[31,32]$. In this context, the gauge/gravity duality is a principle which relates strongly coupled systems to their weak coupled gravity duals. One of the best-studied system is the holographic superconductor. In its simplest form, the gravity sector is a gravitating system with a cosmological constant, a gauge field and a charged scalar field with a potential (for a review see [33]). The dynamics of the system defines a critical temperature above which the system finds itself in its normal phase and the scalar field does not have any dynamics. Below the critical temperature the system undergoes a phase transition to a new configuration. From the gravity side this is interpreted as the black hole to acquire hair while from boundary conformal field theory site this is interpreted as a condensation of the scalar field and the system enters a superconducting phase.

It is well known that all solar system observations, such as light deflection, the perihelion shift of planets, and the gravitational time delay among others, are described within Einstein's General Relativity. The study of geodesics has been performed under several black hole geometries. For instance, see [34-44] for the motion of particles on AdS spacetime. In this work, motivated by black hole solutions with matter fields outside their horizon, we study their effect in the motion of massless and massive particles in the background of a four-dimensional asymptotically AdS black hole with scalar hair [20]. These hairy black holes solutions are characterized by a self-interacting potential that asymptotically tends to the cosmological constant, and the scalar field is regular everywhere outside the event horizon and null at spatial infinity. The geodesics are studied numerically and we discuss the differences in the motion of particles between the four-dimensional asymptotically AdS black holes with scalar hair and their no-hair limit, that is, Schwarzschild AdS black holes. Also, we study classical tests such as perihelion precession, deflection of light and gravitational time delay in order to determine the contribution that arises from the scalar hair.

The paper is organized as follows. In Sect. 2 we give a brief review of the four-dimensional asymptotically AdS black holes with scalar hair that we will consider as background. In Sect. 3 we study the motion of massless and massive par- ticles, and we perform some classical tests such as perihelion precession, deflection of light and gravitational time delay. Finally in Sect. 4 we conclude.

\section{Four-dimensional asymptotically AdS black holes with scalar hair}

The hairy black hole that we consider is solution of the Einstein-Hilbert action with a negative cosmological constant and a neutral scalar field minimally coupled to the curvature having a self-interacting potential $V(\phi)[20]$. The action is given by

$S=\int \mathrm{d}^{4} x \sqrt{-g}\left(\frac{1}{2 \kappa} R-\frac{1}{2} g^{\mu \nu} \nabla_{\mu} \phi \nabla_{\nu} \phi-V(\phi)\right)$,

the self-interacting potential being

$$
\begin{aligned}
V(\phi)= & -F(2+\cosh (\sqrt{2} \phi))+\frac{G}{v^{3}}(6 \sinh (\sqrt{2} \phi) \\
& -2 \sqrt{2} \phi(2+\cosh (\sqrt{2} \phi))) .
\end{aligned}
$$

Here, the cosmological constant is incorporated in the potential, that is, $\Lambda=V(0)(V(0)<0)$. Here $\Lambda=-6 l^{-2} / \kappa, l$ being the length of the AdS space and $\kappa=8 \pi G_{N}$, with $G_{N}$ the Newton constant. This potential has a global maximum at $\phi=0$. The equations of motion are

$R_{\mu \nu}-\frac{1}{2} g_{\mu \nu} R=\kappa T_{\mu \nu}^{(\phi)}$

where the energy momentum tensor $T_{\mu \nu}^{(\phi)}$ for the scalar field is

$T_{\mu \nu}^{(\phi)}=\nabla_{\mu} \phi \nabla_{\nu} \phi-g_{\mu \nu}\left[\frac{1}{2} g^{\rho \sigma} \nabla_{\rho} \phi \nabla_{\sigma} \phi+V(\phi)\right]$,

and the Klein-Gordon equation of the scalar field reads

$\square \phi=\frac{\mathrm{d} V}{\mathrm{~d} \phi}$.

The following metric is a solution of the theory defined by (1):

$\mathrm{d} s^{2}=-f(r) \mathrm{d} t^{2}+f^{-1}(r) \mathrm{d} r^{2}+a^{2}(r) \mathrm{d} \sigma_{k}^{2}$,

where

$$
\begin{aligned}
f(r)= & k+F r(r+v) \\
& +\frac{G}{v^{3}}\left(-v(v+2 r)+2 r(r+v) \ln \left(\frac{r+v}{r}\right)\right),
\end{aligned}
$$

$a^{2}(r)=r(r+v)$,

and the scalar field is

$\phi(r)=\frac{1}{\sqrt{2}} \ln \left(1+\frac{v}{r}\right)$. 
In Eq. (6), $\mathrm{d} \sigma_{k}^{2}$ is the metric of the spatial 2-section, which can have positive, negative or zero curvature, and the coordinates are defined in the ranges $0<r<\infty,-\infty<t<\infty$, $0 \leq \theta<\pi, 0 \leq \phi<2 \pi$. For the lapse function $k=1,0,-1$ parametrizes the curvature of the spatial 2-sections and $F, G$ are constants being proportional to the cosmological constant and to the mass respectively. It was shown that for spherical horizons $k=1$ there is no phase transition of the hairy asymptotically AdS black holes to Schwarzschild AdS black hole. However, for hyperbolic horizons $k=-1$ there exists a phase transition only for negative masses, and the hairy black hole dominates for small temperatures, while for large temperatures the topological black hole would be preferred, for more details see [20].

In the next section we perform a numerical analysis of the geodesics by considering the hairy black hole solution. So, without loss of generality, we consider the following values for the parameters: $k=1, v=-1, F=1$, and $G=2$. Thus, in order to show that these parameters yield a hairy black hole solution we plot in Fig. 1 the behavior of the metric function
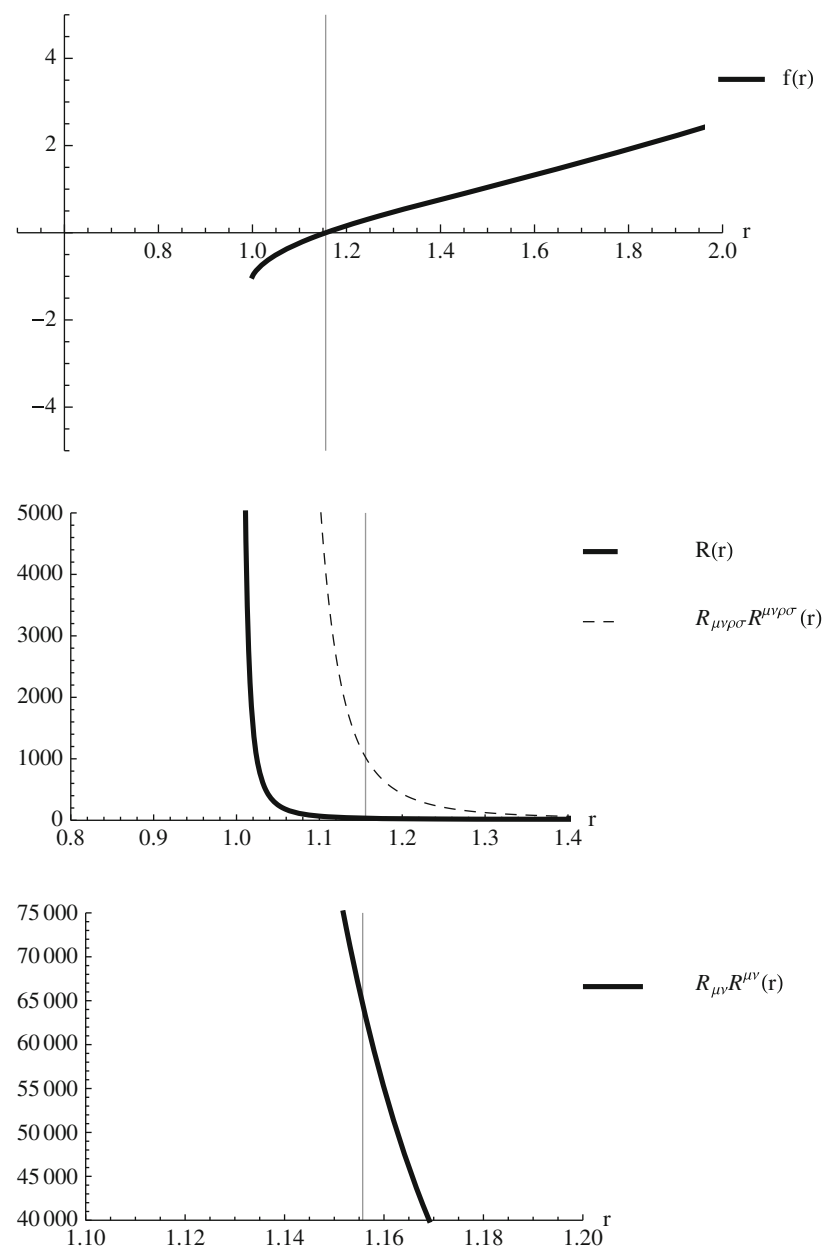

Fig. 1 The behavior of $f(r)$ (top figure), $R(r)$ and $R_{\mu \nu \rho \sigma} R^{\mu v \rho \sigma}(r)$ (center figure) and $R_{\mu \nu} R^{\mu \nu}(r)$ (bottom figure) with $k=1, v=-1$, $F=1$, and $G=2$ $f(r)$, which changes sign for $r=1.15$, signaling the presence of an horizon. Also we plot the behavior of the Ricci scalar $R(r)$, the principal quadratic invariant of the Ricci tensor $R^{\mu \nu} R_{\mu \nu}(r)$, and the Kretschmann scalar $R^{\mu \nu \lambda \tau} R_{\mu \nu \lambda \tau}(r)$, and we observe that there is no Riemann curvature singularity outside the horizon. Also, we observe that the Riemann curvature singularities are covered by the horizon. Therefore, the choice of parameters mentioned above gives a hairy black hole solution which is asymptotically AdS.

\section{Geodesics}

In order to find the geodesics of the spacetime described by (6), we will solve the Euler-Lagrange equations for the variational problem associated with this metric. The Lagrangian associated to the metric (6) is given by

$2 \mathcal{L}=-f(r) \dot{t}^{2}+\frac{\dot{r}^{2}}{f(r)}+a^{2}(r)\left(\dot{\theta}^{2}+\sin ^{2} \theta \dot{\phi}^{2}\right)=-m$,

where $\dot{q}=\mathrm{d} q / \mathrm{d} \tau$, and $\tau$ is an affine parameter along the geodesic that we choose as the proper time. Since the Lagrangian (10) is independent of the cyclic coordinates $(t, \phi)$, their conjugate momenta $\left(\Pi_{t}, \Pi_{\phi}\right)$ are conserved and the equations of motion read

$\dot{\Pi}_{q}-\frac{\partial \mathcal{L}}{\partial q}=0$,

where $\Pi_{q}=\partial \mathcal{L} / \partial \dot{q}$ is the conjugate momentum to the coordinate $q$. The above equation can be written as

$$
\begin{aligned}
& \dot{\Pi}_{t}=0, \quad \dot{\Pi}_{r}=-\frac{\dot{t}^{2}}{2} \frac{\mathrm{d} f(r)}{\mathrm{d} r}+\frac{\dot{r}^{2}}{2} \frac{\mathrm{d} f(r)^{-1}}{\mathrm{~d} r} \\
& +a(r) \frac{\mathrm{d} a(r)}{\mathrm{d} r}\left(\dot{\theta}^{2}+\sin ^{2} \theta \dot{\phi}^{2}\right),
\end{aligned}
$$

which yields

$$
\begin{aligned}
& \Pi_{t}=-f(r) \dot{t}, \quad \Pi_{r}=\frac{\dot{r}}{f(r)}, \\
& \Pi_{\theta}=a^{2} \dot{\theta}, \quad \text { and } \quad \Pi_{\phi}=a^{2} \sin ^{2} \theta \dot{\phi} .
\end{aligned}
$$

Now, without loss of generality, we consider the motion to develop in the invariant plane $\theta=\pi / 2$ and $\dot{\theta}=0$, which is characteristic of the central fields. With this choice, Eqs. (15) and (16) become

$\Pi_{t}=-f(r) \dot{t} \equiv-\sqrt{E}, \quad \Pi_{\phi}=a^{2} \dot{\phi} \equiv L$,

where $E$ and $L$ are dimensionless integration constants associated to each of them. So, inserting Eq. (16) into Eq. (10) we obtain 


$$
\left(\frac{\mathrm{d} r}{\mathrm{~d} \tau}\right)^{2}=E-V(r)
$$

where $V(r)$ is the effective potential given by

$V(r)=f(r)\left[m+\frac{L^{2}}{r(r+v)}\right]$,

where $m$ is the test mass. Finally, by normalization, we shall take $m=1$ for massive particles and $m=0$ for photons.

\subsection{Time-like geodesic}

In order to observe the possible orbits, we plot the effective potential for massive particles (18) which is shown in Fig. (2). In the following, we describe the radial motion and the angular motion.

\subsubsection{Radial motion}

In this case $L=0$. The particles always fall into the horizon from an upper distance determined by the constant of motion $E=30.88$. This fact is due to the attractive force generated by the proportional term to the cosmological constant; see Fig. 2. In Fig. 3 we plot the proper $(\tau)$ and coordinate $(t)$ time as a function of $r$ for a particle falling from a finite distance with zero initial velocity, and we can see that the

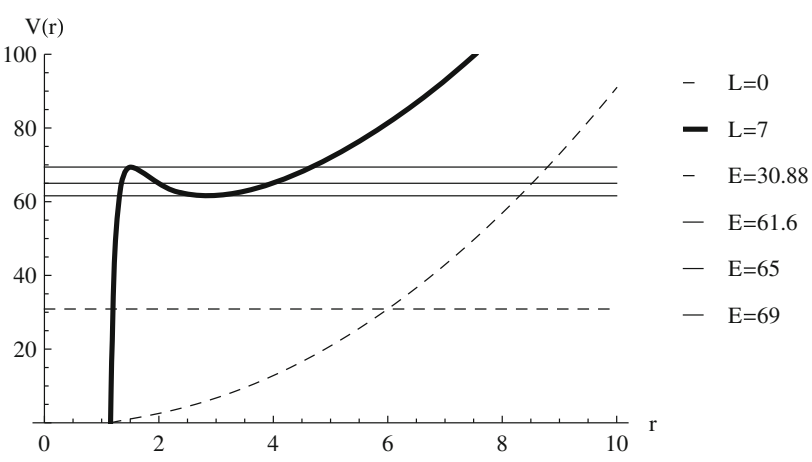

Fig. 2 The behavior of $V(r)$ for radial $(L=7)$ and non-radial $(L=0)$ particles, with $k=1, v=-1, F=1$, and $G=2$

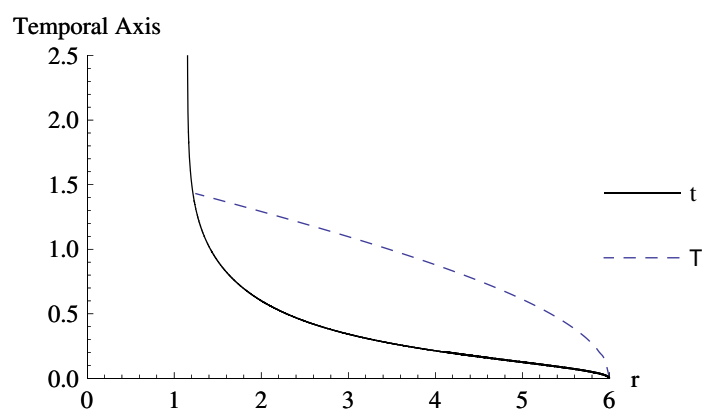

Fig. 3 The behavior of the proper $(\tau)$ and coordinate $(t)$ time as a function of $r$, with $k=1, v=-1, F=1$, and $G=2$ particle falls toward the horizon in a finite proper time. The situation is very different if we consider the trajectory in the coordinate time, where $t$ goes to infinity. This physical result is consistent with the Schwarzschild AdS black hole.

\subsubsection{Angular motion}

For the angular motion we consider $L>0$. The allowed orbits depend on the value of the constant $E$.

- If $E=61.6$ the particle can orbit in a stable circular orbit at $r_{s}=2.84$; see Fig. 2.

- If $E=69.4$ the particle can orbit in an unstable circular orbit at $r_{u}=1.518$. Also, there are two critic orbits that approximates asymptotically to the unstable circular orbit. For the first kind, the particle starts from the rest and a finite distance greater than the unstable radio; see Fig. 4. For the second kind, the particle starts from a finite distance greater than the horizon, but smaller than the unstable radio; see Fig. 4.

- The planetary orbits are constrained to oscillate between an aphelion and a perihelion. We plot in Fig. 5 the planetary orbit for $E=65$. We can observe that the particle completes an oscillation in an angle less than $2 \pi$, contrary to the Schwarzschild AdS black hole, where the angle is greater than $2 \pi[45]$.

It is possible to calculate the periods of the circular orbits $\left(r_{\text {c.o. }}\right)$, which can be stable $\left(r_{\mathrm{s}}\right)$ or unstable $\left(r_{\mathrm{u}}\right)$ orbits using the constant of motion $\sqrt{E}$ and $L$, given by (16), which yields

$T_{\tau}=\frac{2 \pi r_{\text {c.o. }}\left(r_{\text {c.o. }}+v\right)}{L}$,

and

$T_{t}=\frac{2 \pi \sqrt{E} r_{\text {c.o. }}\left(r_{\text {c.o. }}+v\right)}{L f\left(r_{\text {c.o. }}\right)}$,

where $T_{\tau}$ is the period of the orbit with respect to the proper time and $T_{t}$ is the period of the orbit with respect to the coordinate time. It is worth to mention that the periods depend on the value of $v$ and in the limit $v \rightarrow 0$ these periods correspond to the periods of the circular orbits in the spacetime Schwarzschild AdS. On the other hand, for the stable circular orbits it is possible to find the epicycle frequency, given by $\kappa^{2}=V^{\prime \prime}\left(r_{\mathrm{s}}\right) / 2$.

\subsubsection{Perihelion precession}

Here, we follow the treatment performed by Cornbleet [46], which allows us to derive the formula for the advance of the perihelia of planetary orbits. The starting point is to consider the line element in unperturbed Lorentz coordinates 


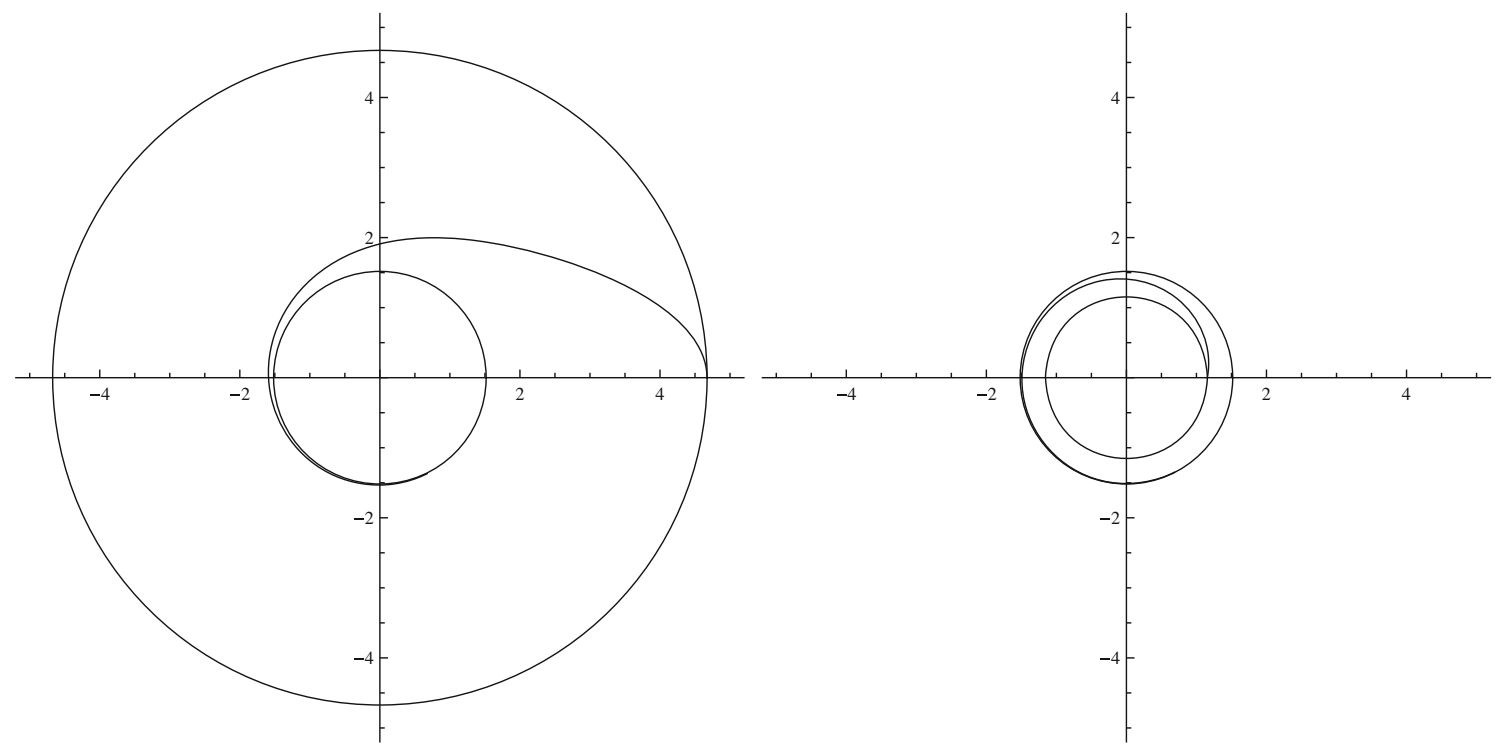

Fig. 4 The first (left figure) and second (right figure) kind of critical orbits with $L=7, k=1, v=-1, F=1$, and $G=2$

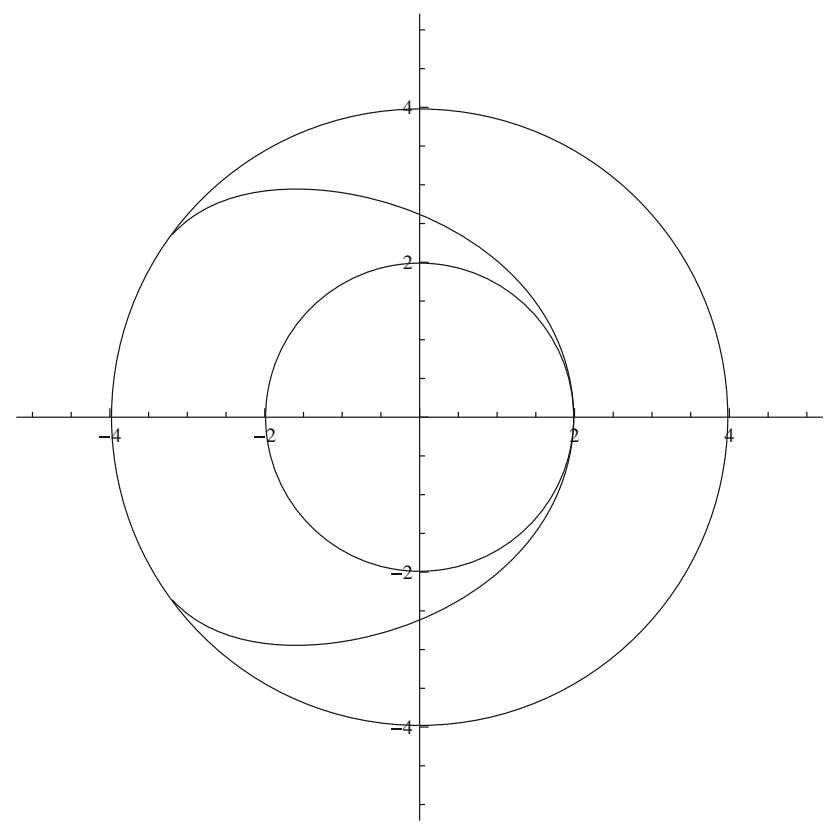

Fig. 5 The planetary orbit with $L=7, k=1, v=-1, F=1$, and $G=2$ for $E=69.4$

$\mathrm{d} s^{2}=-\mathrm{d} t^{2}+\mathrm{d} r^{2}+r^{2}\left(\mathrm{~d} \theta^{2}+\sin ^{2} \theta \mathrm{d} \phi^{2}\right)$,

together with line element (6). So, considering only the radial and time coordinates in the binomial approximation, the transformation gives

$\mathrm{d} \tilde{t} \approx\left(1-\frac{G}{6 r}+\frac{F r^{2}}{2}+\frac{G v}{12 r^{2}}+\frac{F v r}{2}\right) \mathrm{d} t$,
$\mathrm{d} \tilde{r} \approx\left(1+\frac{G}{6 r}-\frac{F r^{2}}{2}-\frac{G v}{12 r^{2}}-\frac{F v r}{2}\right) \mathrm{d} r$.
We will consider two elliptical orbits, one the classical Kepler orbit in $(r, t)$ space and a hairy AdS orbit in $(\tilde{r}, \tilde{t})$ space. Then in the Lorentz space $\mathrm{d} A=\int_{0}^{\mathcal{R}} r \mathrm{~d} r \mathrm{~d} \phi=\mathcal{R}^{2} \mathrm{~d} \phi / 2$, and hence

$\frac{\mathrm{d} A}{\mathrm{~d} t}=\frac{1}{2} \mathcal{R}^{2} \frac{\mathrm{d} \phi}{\mathrm{d} t}$,

which corresponds to Kepler's second law. For the hairy AdS case we have

$\mathrm{d} \tilde{A}=\int_{0}^{\mathcal{R}} a(r) \mathrm{d} \tilde{r} \mathrm{~d} \phi$,

where $\mathrm{d} \tilde{r}$ is given by Eq. (23), and the binomial approximation for the radial function $a(r)$ is

$a(r) \approx r\left(1+\frac{v}{2 r}\right)$.

So, we can write $(25)$ as

$$
\begin{aligned}
\mathrm{d} \tilde{A}= & \int_{0}^{\mathcal{R}} r\left(1+\frac{v}{2 r}\right) \\
& \times\left(1+\frac{G}{6 r}-\frac{F r^{2}}{2}-\frac{G v}{12 r^{2}}-\frac{F v r}{2}\right) \mathrm{d} r \mathrm{~d} \phi \\
\approx & \frac{\mathcal{R}}{2}\left(1+\frac{G}{3 \mathcal{R}}-\frac{F \mathcal{R}^{2}}{4}+\frac{v}{\mathcal{R}}\right) \mathrm{d} \phi .
\end{aligned}
$$

Therefore, applying the binomial approximation we obtain

$$
\begin{aligned}
\frac{\mathrm{d} \tilde{A}}{\mathrm{~d} \tilde{t}}= & \frac{\mathcal{R}}{2}\left(1+\frac{G}{3 \mathcal{R}}-\frac{F \mathcal{R}^{2}}{4}+\frac{v}{\mathcal{R}}\right) \frac{\mathrm{d} \phi}{\mathrm{d} \tilde{t}} \\
\approx & \frac{\mathcal{R}}{2}\left(1+\frac{G}{3 \mathcal{R}}-\frac{F \mathcal{R}^{2}}{4}+\frac{v}{\mathcal{R}}\right) \\
& \times\left(1+\frac{G}{6 \mathcal{R}}-\frac{F \mathcal{R}^{2}}{2}-\frac{G v}{12 \mathcal{R}^{2}}-\frac{F \nu \mathcal{R}}{2}\right) \frac{\mathrm{d} \phi}{\mathrm{d} t} .
\end{aligned}
$$


So, we use this increase to improve the elemental angle from $\mathrm{d} \phi$ to $\mathrm{d} \tilde{\phi}$. Then for a single orbit

$\int_{0}^{\Delta \tilde{\phi}} \mathrm{d} \tilde{\phi}=\int_{0}^{\Delta \phi=2 \pi}\left(1+\frac{G}{2 \mathcal{R}}-\frac{3 F \mathcal{R}^{2}}{4}+\frac{v}{\mathcal{R}}\right) \mathrm{d} \phi$,

where we have neglected products of $G, F$, and $\nu$. The polar form of an ellipse is given by

$\mathcal{R}=\frac{l}{1+\epsilon \cos \phi}$,

where $\epsilon$ is the eccentricity and $l$ is the semi-latus rectum. In this way, plugging Eq. (30) into Eq. (29), we obtain

$$
\begin{aligned}
\Delta \tilde{\phi}= & 2 \pi+\frac{G}{2} \int_{0}^{2 \pi} \frac{1+\epsilon \cos \phi}{l} \mathrm{~d} \phi \\
& -\frac{3 F}{4} \int_{0}^{2 \pi}\left(\frac{l}{1+\epsilon \cos \phi}\right)^{2} \mathrm{~d} \phi \\
& +v \int_{0}^{2 \pi} \frac{1+\epsilon \cos \phi}{l} \mathrm{~d} \phi,
\end{aligned}
$$

which at first order yields

$\Delta \tilde{\phi} \approx 2 \pi+\frac{\pi G}{l}+\frac{3 \pi F l^{2}}{2}+\frac{2 \pi v}{l}$.

Therefore, the perihelion advance has the standard value of general relativity plus the correction term coming from cosmological constant and scalar hair. It is worth to mention that there is a (negative) discrepancy between the observational value of the precession of perihelion for Mercury, $\Delta \tilde{\phi}_{\text {Obs. }}=5599.74 \operatorname{arcsec} /$ Julian - century and the total $\Delta \tilde{\phi}_{\text {Total }}=5603.24 \mathrm{arcsec} /$ Julian - century; see [47]. This may be attributed to the scalar hair correction, given $v=$ $-0.359 \mathrm{~km}$.

\subsection{Null geodesic}

In the next analysis, we consider two kinds of motion, for $L=0$ (radial motion), and $L>0$ (angular motion) of the photons $(m=0)$.

\subsubsection{Radial motion}

In this case, the master equation (17) can be written as

$$
\frac{\mathrm{d} r}{\mathrm{~d} \tau}= \pm \sqrt{E},
$$

where (+) stands for outgoing photons and (-) stands for ingoing photons. The solution of the above equation yields

$$
r= \pm \sqrt{E} \tau+r_{0}
$$

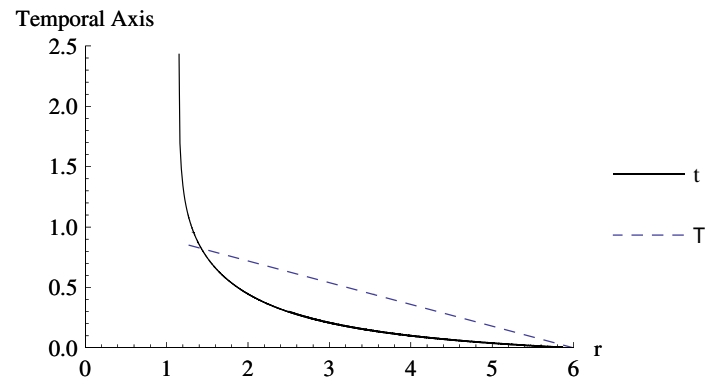

Fig. 6 The behavior of the proper $(\tau)$ and coordinate $(t)$ time for ingoing photons as a function of $r$, with $L=0, k=1, v=-1, F=1$, and $G=2$

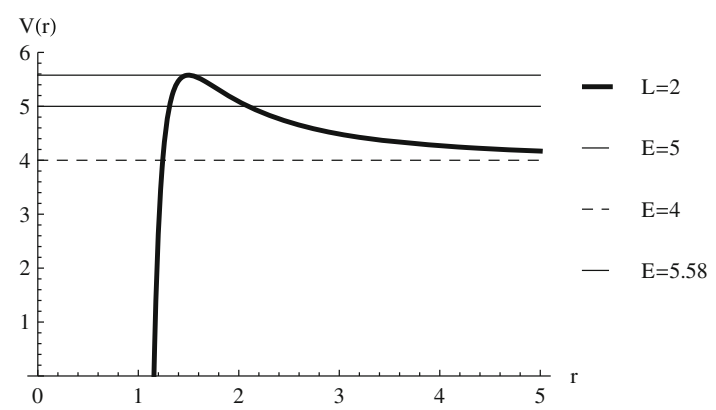

Fig. 7 The behavior of the effective potential for photons as a function of $r$, with $L=2, k=1, v=-1, F=1$, and $G=2$

where $r_{0}$ is an integration constant that corresponds to the initial position of the photon, as in the Schwarzschild AdS case. The photons always fall into the horizon from an upper distance determined by the constant of motion $E=30.88$. In Fig. 6 we plot the proper $(\tau)$ and coordinate $(t)$ time as a function of $r$ for a photon falling from a finite distance $\left(r_{0}=6\right)$, we can see that photons fall toward the horizon in a finite proper time. The situation is very different if we consider the trajectory in the coordinate time, where $t$ goes to infinity.

\subsubsection{Angular motion}

In this case, the allowed orbits for photons depend on the value of the impact parameter $b \equiv L / \sqrt{E}$. Next, based on the impact parameter values shown in Fig. 7, we give a brief qualitative description of the allowed angular motions for photons, described in the following.

- Capture zone: If $0<b<b_{u}$, photons fall inexorably to the horizon, and its cross section, $\sigma$, in this geometry is [48]

$\sigma=\pi b_{u}^{2}$

- Critical trajectories: If $b=b_{u}\left(E_{u}=5.58\right)$, photons can stay in one of the unstable inner circular orbit of radius 


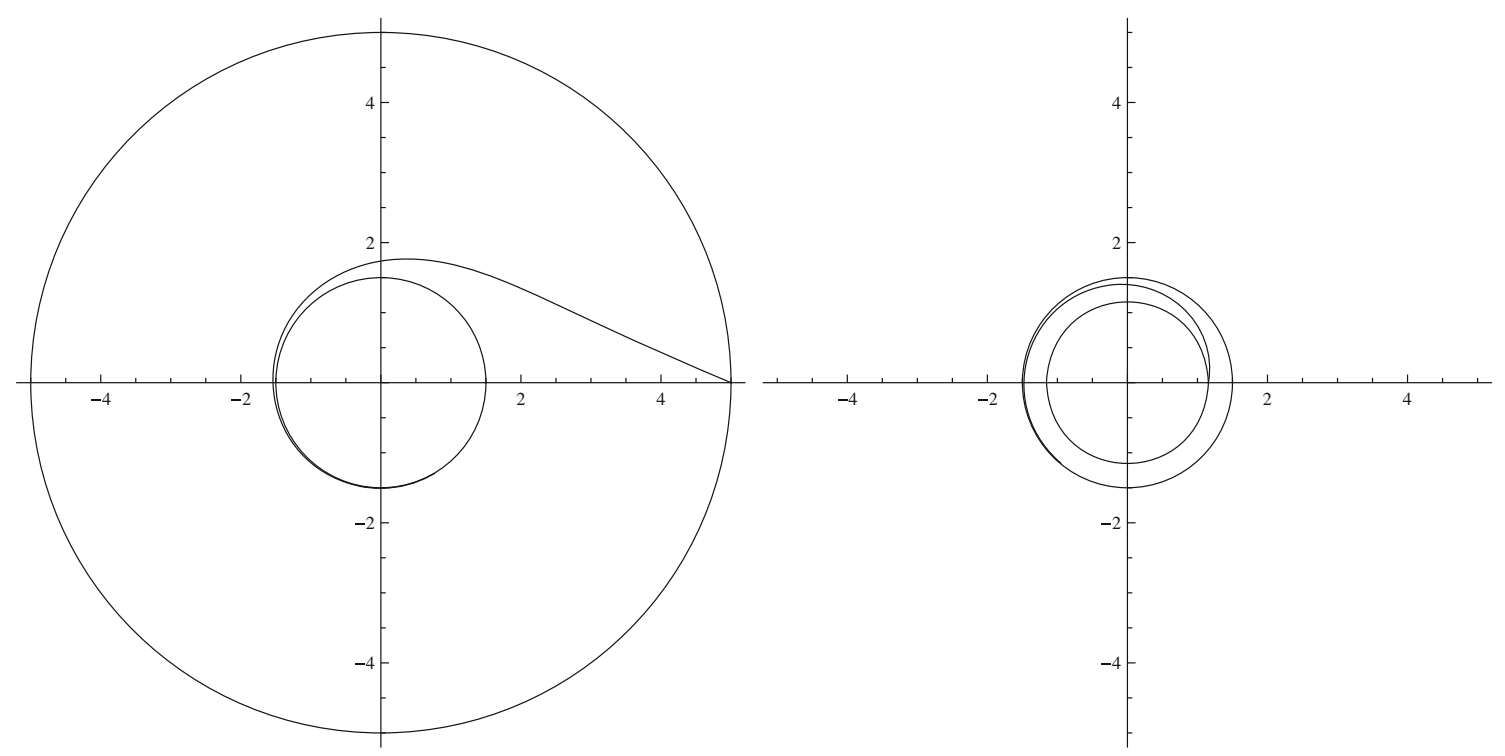

Fig. 8 The first (left figure) and second (right figure) kind of critical orbits with $L=2$ with $k=1, v=-1, F=1$, and $G=2$ for $E=5.58$

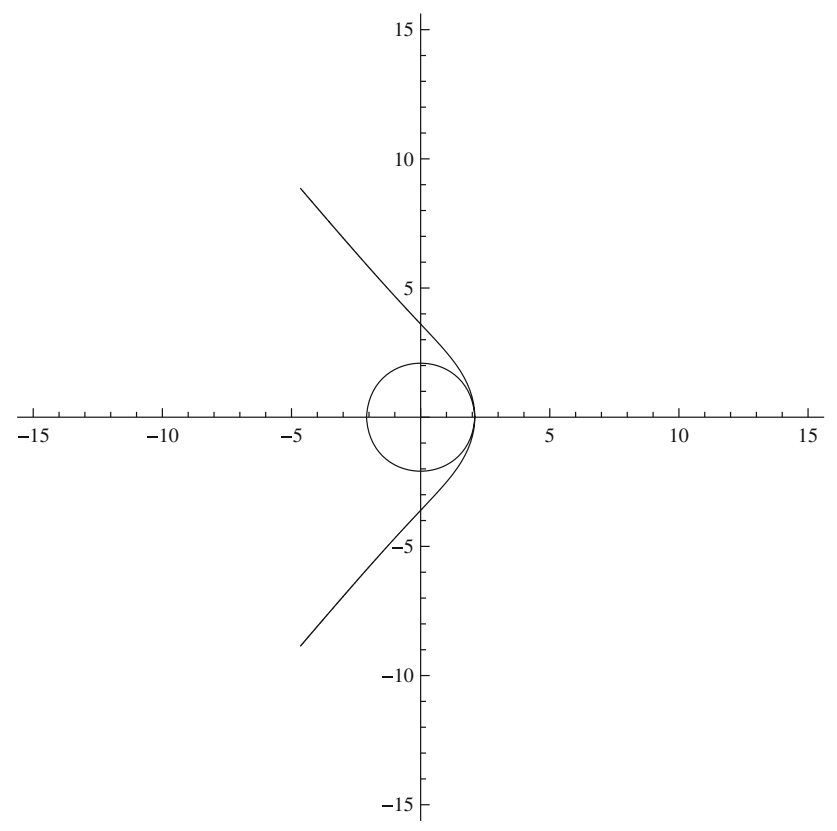

Fig. 9 The deflection of the light with $L=2, k=1, v=-1, F=1$, and $G=2$ for $E=5$

$r_{u}\left(r_{u}=1.5\right)$. Therefore, the photons that arrive from the initial distance $r_{i}\left(r_{+}<r_{i}<r_{u}\right.$, or $\left.r_{u}<r_{i}<\infty\right)$ can asymptotically fall into a circle of radius $r_{u}$; see Fig. 8.

- Deflection zone. If $b_{u}<b<b_{0}=1 / \sqrt{F}$, the photons can fall from infinity to a minimum distance $r_{d}=2.09$ and can go back to infinity. This photons are deflected; see Fig. 9. The other allowed orbits correspond to photons moving into the other side of the potential barrier, which plunge into the singularity. In the next section, we will focus on this topic.

\subsubsection{Deflection of light}

The deflection of light is important because the deflection of light by the Sun is one of the most important tests of general relativity, and the deflection of light by galaxies is the mechanism behind gravitational lenses. The distance of the closest approach, $r_{0}$, for the metric (6) can be defined by

$$
\left(\frac{\mathrm{d} r}{\mathrm{~d} \phi}\right)^{2}=\frac{a(r)^{4}}{b^{2}}-a(r)^{2} f(r),
$$

where $b$ is the impact parameter. Now, by using the change of variables $u=1 / r$, the above equation can be written as

$$
\left(-\frac{\mathrm{d} u}{\mathrm{~d} \phi}\right)^{2}=\frac{1}{b^{2}}(1+v u)^{2}-(1+v u) u^{2} f(u),
$$

which at first order and applying the binomial approximation wherever necessary leads to

$$
\left(-\frac{\mathrm{d} u}{\mathrm{~d} \phi}\right)^{2}=\frac{1}{b^{2}}-F+\frac{2 v u}{b^{2}}-u^{2}+\left(\frac{G}{3}-v\right) u^{3} \text {. }
$$

Following [49] we define

$y \approx u-\frac{v}{b^{2}}-\frac{1}{2}\left(\frac{G}{3}-v\right) u^{2}$.

So, solving for $u$ yields

$u=\frac{v}{b^{2}}+y+\frac{1}{2}\left(\frac{G}{3}-v\right) y^{2}$,

where we have considered the first order terms. Therefore (38) becomes

$\phi(u)=\int_{\left(1 / b^{2}-F\right)^{-1 / 2}}^{\infty} \frac{1+\left(\frac{G}{3}-v\right) y}{\sqrt{\frac{1}{b^{2}}-F-y^{2}}} \mathrm{~d} y$. 
This can be integrated to give

$\phi_{\infty}=\frac{\pi}{2}+\left(\frac{G}{3}-v\right) \sqrt{\frac{1}{b^{2}}-F}$,

and by considering $\Delta \phi=2 \phi_{\infty}-\pi$ (see Fig. 9), it is possible to find the deflection angle accurately, and it reads

$\Delta \phi=2\left(\frac{G}{3}-v\right) \sqrt{\frac{1}{b^{2}}-F}$.

Therefore, the deflection of light is given by the standard value plus the correction term coming from the cosmological constant $(F)$ and scalar hair $(v)$. It is worth to mention that there is a discrepancy between the theoretical value and the observational value of deflection light measured by Eddington and Dyson in the solar eclipse of March 29, 1919. For the Sobral expedition this value is $\Delta \phi_{\text {Obs. }}=1.98 \pm 0.16^{\prime \prime}$, and it is $\Delta \phi_{\text {Obs. }}=1.61 \pm 0.40^{\prime \prime}$ for the Principe expedition [50]. Currently, the mean value is $\Delta \phi_{\text {Obs. }}=1.89$ [51]. So, by attributing this discrepancy to the scalar hair correction and neglecting the contribution from the cosmological constant, one finds $v=-0.386 \mathrm{~km}$, for $\Delta \phi_{\text {Obs. }}=1.98$, and $v=-0.235 \mathrm{~km}$, for the mean value of $\Delta \phi_{\text {Obs. }}$.

\subsubsection{Gravitational time delay}

An interesting relativistic effect in the propagation of light rays is the apparent delay in the time of propagation for a light signal passing near the Sun, which is a relevant correction for astronomic observations, and is called the Shapiro time delay. The time delay of Radar Echoes corresponds to the determination of the time delay of radar signals which are transmitted from the Earth through a region near the Sun to another planet or spacecraft and then reflected back to the Earth. The time interval between emission and return of a pulse as measured by a clock on the Earth is

$t_{12}=2 t\left(r_{1}, \rho_{0}\right)+2 t\left(r_{2}, \rho_{0}\right)$,

where $\rho_{0}$ is the closest approach to the Sun. Now, in order to calculate the time delay we use (17) and the coordinate time,

$\dot{r}=\dot{\mathrm{d} r} \frac{\sqrt{E}}{\mathrm{~d} t}=\frac{\mathrm{d} r}{f(r)} \frac{\mathrm{d} t}{.}$

So, (17) can be written as

$\frac{\sqrt{E}}{f(r)} \frac{\mathrm{d} r}{\mathrm{~d} t}=\sqrt{E-\frac{L^{2}}{a(r)^{2}} f(r)}$.
By considering $\rho_{0}$, the closest approach to the Sun, $\mathrm{d} r / \mathrm{d} t$ vanishes, so that

$\frac{E}{L^{2}}=\frac{f\left(\rho_{0}\right)}{a\left(\rho_{0}\right)^{2}}$.

Now, by inserting (47) in (46), the coordinate time which the light requires to go from $\rho_{0}$ to $r$ is

$t\left(r, \rho_{0}\right)=\int_{\rho_{0}}^{r} \frac{\mathrm{d} r}{f(r) \sqrt{1-\frac{a\left(\rho_{0}\right)^{2}}{f\left(\rho_{0}\right)} \frac{f(r)}{a(r)^{2}}}}$.

So, at first order correction we obtain

$$
\begin{aligned}
t\left(r, \rho_{0}\right)= & \sqrt{r^{2}-\rho_{0}^{2}}+\frac{G}{3} \ln \left(\frac{r+\sqrt{r^{2}-\rho_{0}^{2}}}{\rho_{0}}\right) \\
& +\frac{1}{2}\left(\frac{G}{3}+v\right) \sqrt{\frac{r-\rho_{0}}{r+\rho_{0}}} \\
& -\frac{F}{6} \sqrt{r^{2}-\rho_{0}^{2}}\left(2 r^{2}+\rho_{0}^{2}\right) .
\end{aligned}
$$

Therefore, for the circuit from point 1 to point 2 and back, the delay in the coordinate time is

$$
\begin{aligned}
\Delta t & :=2\left[t\left(r_{1}, \rho_{0}\right)+t\left(r_{2}, \rho_{0}\right)-\sqrt{r_{1}^{2}-\rho_{0}^{2}}-\sqrt{r_{2}^{2}-\rho_{0}^{2}}\right] \\
& =t_{G}+t_{F}+t_{\nu}
\end{aligned}
$$

where

$\begin{aligned} t_{G}= & \frac{G}{3}\left[2 \ln \left(\frac{\left(r_{1}+\sqrt{r_{1}^{2}-\rho_{0}^{2}}\right)\left(r_{2}+\sqrt{r_{2}^{2}-\rho_{0}^{2}}\right)}{\rho_{0}^{2}}\right)\right. \\ & \left.+\sqrt{\frac{r_{1}-\rho_{0}}{r_{1}+\rho_{0}}}+\sqrt{\frac{r_{2}-\rho_{0}}{r_{2}+\rho_{0}}}\right], \\ t_{F}= & -\frac{F}{6}\left[\sqrt{r_{1}^{2}-\rho_{0}^{2}}\left(2 r_{1}^{2}+\rho_{0}^{2}\right)+\sqrt{r_{2}^{2}-\rho_{0}^{2}}\left(2 r_{2}^{2}+\rho_{0}^{2}\right)\right],\end{aligned}$

$t_{v}=v\left(\sqrt{\frac{r_{1}-\rho_{0}}{r_{1}+\rho_{0}}}+\sqrt{\frac{r_{2}-\rho_{0}}{r_{2}+\rho_{0}}}\right)$.

For a round trip in the solar system, we have $\left(\rho_{0}<<r_{1}, r_{2}\right)$

$\Delta t \approx \frac{2 G}{3} \ln \left(\frac{4 r_{1} r_{2}}{\rho_{0}}\right)+2\left(\frac{G}{3}+v\right)-\frac{F}{3}\left(r_{1}^{3}+r_{2}^{3}\right)$

Therefore, as in the previous cases the time delay has the standard value of general relativity plus the correction term coming from the cosmological constant and scalar hair. 


\section{Concluding comments}

We have considered a four-dimensional asymptotically AdS black hole with scalar hair [20]. These solutions asymptotically give the Schwarzschild anti-de Sitter solution, and they are characterized by a scalar field with a logarithmic behavior, being regular everywhere outside the event horizon and null at spatial infinity, and by a self-interacting potential, which tends to the cosmological constant at spatial infinity. The equations for the geodesics were solved numerically in order to study their behavior. We note that radial motion as a result is found to be equivalent to the Schwarzschild AdS spacetime [45]. Mainly, we have found that it is possible to find bounded orbits like planetary orbits in the background of a four-dimensional asymptotically AdS black holes with scalar hair. However, the periods associated to circular orbits are modified by the presence of the scalar hair. Besides, we have found that some classical tests such as perihelion precession, deflection of light, and gravitational time delay have the standard value of general relativity plus a correction term coming from the cosmological constant and scalar hair. Finally, we found a specific value of the parameter associated to the scalar hair, in order to explain the discrepancy between the theory and the observations, for the perihelion precession of Mercury $(v=-0.359 \mathrm{~km})$ and light deflection $\left(v=-0.386 \mathrm{~km}\right.$ for $\left.\Delta \phi_{\text {Obs. }}=1.98\right)$. Interestingly, these values are of the same order and sign. In furthering our understanding, it would be interesting to study the motion of massless and massive particles in a charged hairy black hole. Work in this direction is in progress.

Acknowledgments This work was funded by Comisión Nacional de Ciencias y Tecnología through FONDECYT Grants 11140674 (PAG) and $11121148(\mathrm{YV})$.

Open Access This article is distributed under the terms of the Creative Commons Attribution 4.0 International License (http://creativecomm ons.org/licenses/by/4.0/), which permits unrestricted use, distribution, and reproduction in any medium, provided you give appropriate credit to the original author(s) and the source, provide a link to the Creative Commons license, and indicate if changes were made.

Funded by SCOAP ${ }^{3}$.

\section{References}

1. N. Bocharova, K. Bronnikov, V. Melnikov, Vestn. Mosk. Univ. Fiz. Astron. 6, 706 (1970)

2. J.D. Bekenstein, Ann. Phys. 82, 535 (1974)

3. J.D. Bekenstein, Black holes with scalar charge. Ann. Phys. 91, 75 (1975)

4. K.A. Bronnikov, Y.N. Kireyev, Instability of black holes with scalar charge. Phys. Lett. A 67, 95 (1978)

5. K.G. Zloshchastiev, On co-existence of black holes and scalar field. Phys. Rev. Lett. 94, 121101 (2005). arXiv:hep-th/0408163
6. T. Torii, K. Maeda, M. Narita, No-scalar hair conjecture in asymptotic de Sitter spacetime. Phys. Rev. D 59, 064027 (1999). arXiv:gr-qc/9809036

7. C. Martinez, R. Troncoso, J. Zanelli, De Sitter black hole with a conformally coupled scalar field in four-dimensions. Phys. Rev. D 67, 024008 (2003). arXiv:hep-th/0205319

8. T.J.T. Harper, P.A. Thomas, E. Winstanley, P.M. Young, Instability of a four-dimensional de Sitter black hole with a conformally coupled scalar field. Phys. Rev. D 70, 064023 (2004) arXiv:gr-qc/0312104

9. G. Dotti, R.J. Gleiser, C. Martinez, Static black hole solutions with a self interacting conformally coupled scalar field. Phys. Rev. D 77, 104035 (2008). arXiv:0710.1735 [hep-th]

10. T. Torii, K. Maeda, M. Narita, Scalar hair on the black hole in asymptotically anti-de Sitter spacetime. Phys. Rev. D 64, 044007 (2001)

11. E. Winstanley, On the existence of conformally coupled scalar field hair for black holes in (anti-)de Sitter space. Found. Phys. 33, 111 (2003). arXiv:gr-qc/0205092

12. C. Martinez, R. Troncoso, J. Zanelli, Exact black hole solution with a minimally coupled scalar field. Phys. Rev. D 70, 084035 (2004). arXiv:hep-th/0406111

13. C. Martinez, J.P. Staforelli, R. Troncoso, Topological black holes dressed with a conformally coupled scalar field and electric charge. Phys. Rev. D 74, 044028 (2006). arXiv:hep-th/0512022

14. C. Martinez, R. Troncoso, Electrically charged black hole with scalar hair. Phys. Rev. D 74, 064007 (2006). arXiv:hep-th/0606130

15. T. Kolyvaris, G. Koutsoumbas, E. Papantonopoulos, G. Siopsis, A new class of exact hairy black hole solutions. Gen. Rel. Grav. 43, 163 (2011). arXiv:0911.1711 [hep-th]

16. A. Anabalon, Exact black holes and universality in the backreaction of non-linear sigma models with a potential in (A)dS4. JHEP 1206, 127 (2012). arXiv:1204.2720 [hep-th]

17. A. Anabalon, J. Oliva, Exact hairy black holes and their modification to the universal law of gravitation. Phys. Rev. D 86, 107501 (2012). arXiv:1205.6012 [gr-qc]

18. A. Anabalon, A. Cisterna, Asymptotically (anti) de Sitter black holes and wormholes with a self interacting scalar field in four dimensions. Phys. Rev. D 85, 084035 (2012). arXiv:1201.2008 [hep-th]

19. Y. Bardoux, M.M. Caldarelli, C. Charmousis, Conformally coupled scalar black holes admit a flat horizon due to axionic charge. JHEP 1209, 008 (2012). arXiv:1205.4025 [hep-th]

20. P.A. Gonzalez, E. Papantonopoulos, J. Saavedra, Y. Vasquez, Fourdimensional asymptotically AdS black holes with scalar hair. JHEP 1312, 021 (2013). arXiv:1309.2161 [gr-qc]

21. P.A. Gonzalez, E. Papantonopoulos, J. Saavedra, Y. Vasquez, Extremal hairy black holes. JHEP 1411, 011 (2014). arXiv: 1408.7009 [gr-qc]

22. L. Amendola, Cosmology with nonminimal derivative couplings. Phys. Lett. B 301, 175 (1993). arXiv:gr-qc/9302010

23. S.V. Sushkov, Exact cosmological solutions with nonminimal derivative coupling. Phys. Rev. D 80, 103505 (2009). arXiv:0910.0980 [gr-qc]

24. T. Kolyvaris, G. Koutsoumbas, E. Papantonopoulos, G. Siopsis, Scalar hair from a derivative coupling of a scalar field to the Einstein tensor. Class. Quant. Grav. 29, 205011 (2012). arXiv:1111.0263 [gr-qc]

25. T. Kolyvaris, G. Koutsoumbas, E. Papantonopoulos, G. Siopsis, Phase transition to a hairy black hole in asymptotically flat spacetime. arXiv: 1308.5280 [hep-th]

26. L. Sadeghian, C.M. Will, Testing the black hole no-hair theorem at the galactic center: perturbing effects of stars in the surrounding cluster. Class. Quant. Grav. 28, 225029 (2011). arXiv:1106.5056 [gr-qc] 
27. E. Berti, V. Cardoso, L. Gualtieri, M. Horbatsch, U. Sperhake, Numerical simulations of single and binary black holes in scalartensor theories: circumventing the no-hair theorem. Phys. Rev. D 87, 124020 (2013). arXiv:1304.2836 [gr-qc]

28. C.A.R. Herdeiro, E. Radu, Kerr black holes with scalar hair. Phys. Rev. Lett. 112, 221101 (2014). arXiv:1403.2757 [gr-qc]

29. C. Herdeiro, E. Radu, Construction and physical properties of Kerr black holes with scalar hair. Class. Quant. Grav. 32(14), 144001 (2015). arXiv:1501.04319 [gr-qc]

30. S.A. Hartnoll, Lectures on holographic methods for condensed matter physics. Class. Quant. Grav. 26, 224002 (2009). arXiv:0903.3246 [hep-th]

31. S.S. Gubser, Phase transitions near black hole horizons. Class. Quant. Grav. 22, 5121 (2005). arXiv:hep-th/0505189

32. S.S. Gubser, Breaking an Abelian gauge symmetry near a black hole horizon. Phys. Rev. D 78, 065034 (2008). arXiv:0801.2977 [hep-th]

33. G.T. Horowitz, Theory of superconductivity. Lect. Notes Phys. 828, 313 (2011). arXiv:1002.1722 [hep-th]

34. Z. Stuchlik, The motion of test particles in black-hole backgrounds with non-zero cosmological constant. Bull. Astronom. Inst. Czechoslov. 34(3), 129-149 (1983)

35. Z. Stuchlik, S. Hledik, Some properties of the Schwarzschild-de Sitter and Schwarzschild-anti-de Sitter space-times. Phys. Rev. D 60, 044006 (1999)

36. Z. Stuchlik, S. Hledik, Equatorial photon motion in the KerrNewman spacetimes with a non-zero cosmological constant. Class. Quant. Grav. 17, 4541 (2000). arXiv:0803.2539 [gr-qc]

37. N. Cruz, M. Olivares, J.R. Villanueva, The geodesic structure of the Schwarzschild anti-de Sitter black hole. Class. Quant. Grav. 22, 1167 (2005). arXiv:gr-qc/0408016

38. M. Vasudevan, K.A. Stevens, Integrability of particle motion and scalar field propagation in Kerr-(anti) de Sitter black hole spacetimes in all dimensions. Phys. Rev. D 72, 124008 (2005). arXiv:gr-qc/0507096

39. E. Hackmann, C. Lammerzahl, Geodesic equation in Schwarzschild-(anti-) de Sitter space-times: analytical solutions and applications. Phys. Rev. D 78, 024035 (2008). arXiv:1505.07973 [gr-qc]
40. E. Hackmann, C. Lammerzahl, Complete analytic solution of the geodesic equation in Schwarzschild-(anti-) de Sitter spacetimes. Phys. Rev. Lett. 100, 171101 (2008). arXiv:1505.07955 [gr-qc]

41. M. Olivares, J. Saavedra, J.R. Villanueva, C. Leiva, Motion of charged particles on the Reissner-Nordstróm (anti)-de Sitter black holes. Mod. Phys. Lett. A 26, 2923 (2011). arXiv:1101.0748 [grqc]

42. N. Cruz, M. Olivares, J. Saavedra, J.R. Villanueva, Null geodesics in the Reissner-Nordstrom anti-de Sitter black holes. arXiv:1111.0924 [gr-qc]

43. A. Larranaga, Geodesic structure of the noncommutative Schwarzschild anti-de Sitter black hole I: timelike geodesics. Rom. J. Phys. 58, 50 (2013). arXiv:1110.0778 [gr-qc]

44. J.R. Villanueva, J. Saavedra, M. Olivares, N. Cruz, Photons motion in charged anti-de Sitter black holes. Astrophys. Space Sci. 344, 437 (2013)

45. S. Chandrasekhar, The Mathematical Theory of Black Holes (Oxford University Press, New York, 1983)

46. S. Cornbleet, Elementary derivation of the advance of the perihelion of a planetary orbit. Am. J. Phys. 61, 650 (1993)

47. M. Olivares, J.R. Villanueva, Massive neutral particles on heterotic string theory. Eur. Phys. J. C 73, 2659 (2013). arXiv:1311.4236 [grqc]

48. R.M. Wald, General Relativity (The University Chicago Press, Chicago, 1984)

49. B. Shutz, A First Course in General Relativity (Cambridge University Press, New York, 2009)

50. N. Straumann, General Relativity and Relativistic Astrophysics (Springer, Berlin, 1984)

51. L. Huang, F. He, H. Huang, M. Yao, The gravitational deflection of light in F(R)-gravity. Int. J. Theor. Phys. 53, 1947 (2014). arXiv:1303.3662 [gr-qc] 